\title{
An Optical Conveyor for Molecules
}

\author{
Franz M. Weinert and Dieter Braun*
}

\author{
Systems Biophysics, Center for Nanoscience, Physics Department, Ludwig Maximilians \\ Universität München, Amalienstrasse 54, 80799 München, Germany
}

Received August 2, 2009; Revised Manuscript Received September 21, 2009

\begin{abstract}
Trapping single ions under vacuum allows for precise spectroscopy in atomic physics. The confinement of biological molecules in bulk water is hindered by the lack of comparably strong forces. Molecules have been immobilized to surfaces, however often with detrimental effects on their function. Here, we optically trap molecules by creating the microscale analogue of a conveyor belt: a bidirectional flow is combined with a perpendicular thermophoretic molecule drift. Arranged in a toroidal geometry, the conveyor accumulates a hundredfold excess of 5-base DNA within seconds. The concentrations of the trapped DNA scale exponentially with length, reaching trapping potential depths of $14 k T$ for 50 bases. The mechanism does not require microfluidics, electrodes, or surface modifications. As a result, the trap can be dynamically relocated. The optical conveyor can be used to enhance diffusion-limited surface reactions, redirect cellular signaling, observe individual biomolecules over a prolonged time, or approach single-molecule chemistry in bulk water.
\end{abstract}

At small scales, Brownian motion dominates the movement of small biomolecules, which renders their handling and positioning a difficult task in free solution. ${ }^{1-4}$ While the relaxation time scale of mass diffusion on the micrometer scale is on the order of milliseconds, heat diffusion of water is still typically 3 orders of magnitude faster. Our idea here is to use these faster thermal fields to confine and control the position of molecules despite their thermal motion.

Thermophoresis moves molecules by a temperature gradient. ${ }^{5-8}$ This effect is weak for small molecules but can be amplified by a bidirectional flow of the fluid perpendicular to the gradient. ${ }^{9}{ }^{910}$ As a result, the molecules are pushed by thermophoresis into one side of the flow and are transported preferentially in one direction. Applied to an axially symmetric geometry, freely diffusing molecules in a fluid film are expected to accumulate to a single spot from all directions (Figure 1a). In effect, thermophoresis pushes the molecules upward where the water flow shuttles them to the center.

Interestingly, this intricate situation of counterflow and thermal gradient can be created solely by optical means. The thermal gradient is applied by focused infrared absorption of a $\mathrm{Cr}$ film below the water (Figure 1b). The bidirectional flow is generated by a moving laser spot, based on the recently shown thermoviscous fluid pump which uses thermal expansion ${ }^{11}$ in thermally created viscosity gradients. ${ }^{12,13} \mathrm{We}$ impose an outward flow near the bottom warm side by a radial laser pattern. The counterflow at the top is ensured by mass conservation. This situation allows the generation of a bidirectional flow in very thin fluid layers of only $2 \mu \mathrm{m}$. With a mean temperature difference of $15 \mathrm{~K}$ and a diameter of 200 $\mu \mathrm{m}$, this conveyor belt becomes very efficient even for small

\footnotetext{
* Corresponding author, dieter.braun@1mu.de.
}

polymers with lengths down to $1.5 \mathrm{~nm}$. Figure 1c shows singlestranded oligonucleotides with lengths of 5, 10, and 50 bases, trapped within $3 \mathrm{~s}$. The longer DNA molecules are accumulated to higher concentrations and are more tightly confined in the center, with an 1/e decay at a radius of 14 $\mu \mathrm{m}$. By inverting the flow, the conveyor transports the molecules equally efficiently in an outward direction as shown for 1000 base pair DNA in Figure 1c, fourth image.

The physics of the conveyor belt can be simulated using finite elements (Femlab, Comsol). In an axial symmetric geometry we model a bidirectional flow with a maximal speed $v_{\max }=200 \mu \mathrm{m} / \mathrm{s}^{12,13}$ within the trapping region $r<$ $100 \mu \mathrm{m}$. We add the thermophoretic drift $v=S_{\mathrm{T}} D \nabla T$ into the diffusion equation with a temperature gradient $\nabla T$ matching temperature imaging measurements using the temperature-sensitive dye Cy5. ${ }^{13}$ The optical conveyor is simulated for various DNA lengths. Values for the diffusion coefficient $D$ and the Soret coefficient $S_{\mathrm{T}}$ are phenomenologically interpolated to match measurements (see Methods). Figure $2 \mathrm{a}$ shows the simulated concentration for 50 base DNA (color scale). The simulation predicts a $\left(2 \times 10^{6}\right)$-fold increase of the center concentration in the steady state.

The trapping mechanism transports the particles into the center within $3 \mathrm{~s}$. After that period the relative concentration profile inside the trap is equilibrated. To further increase the absolute value of the center concentration, molecules have to diffuse into the region of the trap. To fill the trap up to its steady-state concentration, all molecules within a radius of $10 \mathrm{~mm}$ would be needed to enter the trap. Figure $1 \mathrm{~b}$ shows the measured time trace of the center concentration, compared to the simulation prediction. The two different regimes can be clearly distinguished. 

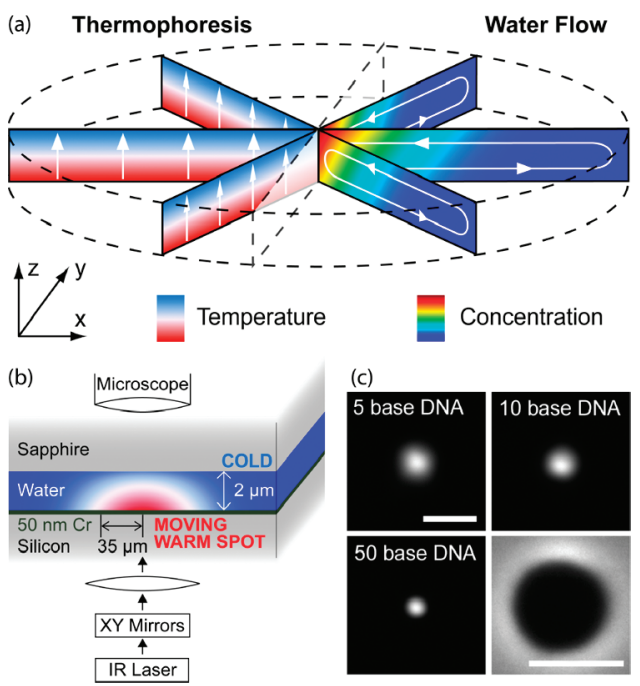

Figure 1. Conveyor trap. (a) Biomolecules are efficiently accumulated by a combination of a temperature gradient and perpendicular bidirectional flow. Since thermophoresis predominantly locates the molecules at the colder top, the conveyor-like water flow transports the molecules to the center. (b) Both the temperature gradient and the water flow are generated by a spot of enhanced temperature at the bottom, induced by the laser absorption in a thin metal layer. The water flow is the result of the coaxial thermoviscous pumping in radial direction. (c) Short DNA is trapped from a diameter of $200 \mu \mathrm{m}$ within $3 \mathrm{~s}$. Longer DNA shows a stronger confinement. The single-stranded DNA oligos with lengths of 5,10 , and 50 bases are labeled with the fluorescent dye

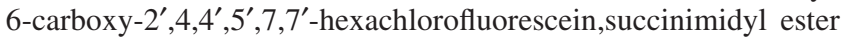
(HEX) to visualize the concentration. By reversal of the fluid flow, the molecules are transported off the conveyor, shown by 1000 base pair DNA stained with the intercalating dye TOTO-1. Scale bars are $100 \mu \mathrm{m}$.

The effective potential depth of the trap can be calculated by assuming a Boltzmann distribution of the DNA molecules in steady state: $U(r)=-k T \ln \left(c / c_{0}\right)$. Figure $2 \mathrm{c}$ shows the simulated shapes of the potentials for $50,22,10$, and 5 base DNA. Five base DNA is expected to be trapped with a potential of $5 k T$ referring to a 200 -fold accumulation. The linear-shaped potential results in an exponential distribution of the concentration over the radius of the trap.

The radial fluorescence intensity of trapped DNA molecules is shown in Figure 2d. The intensities are normalized to the center concentration to compare the accumulation efficiency by the confinement of the distribution for different polymer lengths. The concentration profile differs only in the center of the trap from the expected exponential shape. This is explained by the size of the thermal laser spot with a diameter of $d_{1 / e}=70 \mu \mathrm{m}$, which is used to drive the bidirectional fluid flow. In the center the laser spot overlaps and cancels out the flow. This lowers the efficiency of the trap in the center region.

The radial distribution of the measured intensity is fitted with an exponential function in the region beyond $r=10$ $\mu \mathrm{m}$. The trapping potential is obtained from the intensity ratio $U_{\mathrm{TRAP}} / k T=-\ln \left(c / c_{0}\right)=-\ln \left[I_{\mathrm{EXP}}(0) / I_{\mathrm{FIT}}(100 \mu \mathrm{m})\right]$, where $I_{\operatorname{EXP}}(0)$ is the measured fluorescence intensity in the center. We take the extrapolated intensity at the edge of the trapping region $I_{\mathrm{FIT}}(100 \mu \mathrm{m})$ where the fluorescence measurement is limited by background and the dynamic range of the camera. Figure 2e shows the measured potential depths of the molecule conveyor for single-stranded DNA molecules of different lengths (open circles). The simulated data (solid line) describes the experimental results quantitatively.

Since the conveyor belt is driven entirely optically, its position can be changed easily. Instead of waiting for the molecules to diffuse into the trap, they can be collected while passing over them. Figure 3 a shows $40 \mathrm{~nm}$ polystyrene beads. Comparable to a vacuum cleaner, the radial conveyor belt collects all particles on the trace (movie in Supporting Information). A similar protocol traps 50 base DNA molecules (Figure 3b, movie in Supporting Information).

The shown optofluidic approach allows the assembly of molecules with sizes on the order of a few nanometers from an area of hundreds of micrometers. The principle is related to thermogravitational columns $\mathrm{s}^{14,15}$ which were initially used to separate isotopes in gases. ${ }^{16}$ While thermogravitiational columns drive the fluid flow by gravity, we enforce the flow by thermoviscous expansion. ${ }^{12,13}$ As a result, we shrink the geometry 100 -fold, leading to 10000 -fold faster equilibration times.

Notably, the efficiency of the conveyor does not depend on the temperature gradient. Similar to thermogravitational columns, ${ }^{15}$ the temperature difference $\Delta T$, the aspect ratio of the chamber $r$ (i.e. the trapping radius divided by the thickness) together with the Soret coefficient $S_{\mathrm{T}}$ determines exponentially the maximal concentration enhancement $c / c_{0}=\exp \left[0.3 S_{\mathrm{T}} \Delta T r\right]$. The prefactor 0.3 was determined from the simulation. To work at this limit, the flow speed of the conveyor has to be balanced against the thermophoretic relaxation time of the molecules in the z-direction. However the fluid flow in the conveyor raises quadratically with temperature ${ }^{12,13}$ due to its optothermal driving as shown in Figure $4 \mathrm{a}$. Therefore, the efficiency of the conveyor can actually drop for increased temperature differences. We plot the simulated steady-state concentration of the conveyor in Figure $4 \mathrm{~b}$. While we see that the faster flow increases the trapping of smaller molecules at higher temperature differences, the accumulation drops for the case of 50 base DNA as result of a nonoptimal fluid flow speed. The onset of the accumulation becomes hyperexponential at small temperatures since the flow velocity is too slow for an optimal horizontal transport of the molecules (inset Figure 4b).

To trap smaller molecules such as salts or single fluorescence dyes, one can for example increase the radius of the trap and exponentially raise the steady-state molecule concentration in its center. Also, as seen in Figure 4b, the fluid speed reached by the conveyor is not yet optimal for the shorter DNA probes at the given temperature difference of $15 \mathrm{~K}$. However, the conveyor becomes more efficient for thinner chambers at a constant radius. For example, a 10fold thinner chamber $(200 \mathrm{~nm})$ requires a 10 -fold faster convection flow which can be triggered by an equally 10fold faster scanner. ${ }^{13}$ As a result, the trapping potential depth is enhanced 10-fold and the steady-state concentration of 5 bases of DNA for example would raise from 200-fold to hypothetical $10^{23}$-fold levels. Such conditions would induce a potential for a single fluorescent chromophore with a depth 
(a) Finite Element Simulation

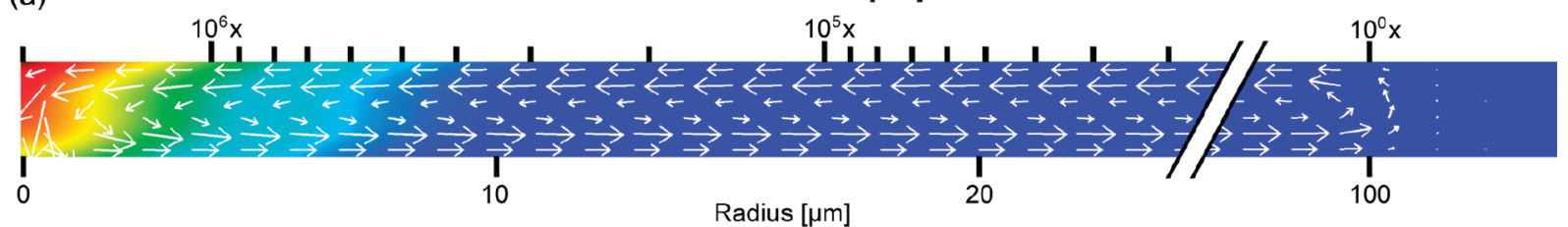

(b)

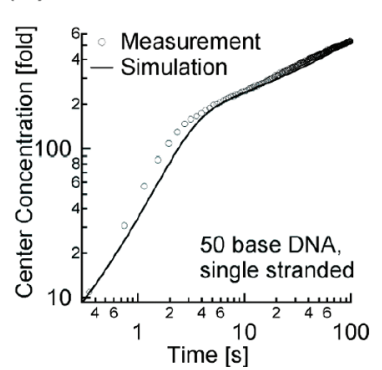

(c)

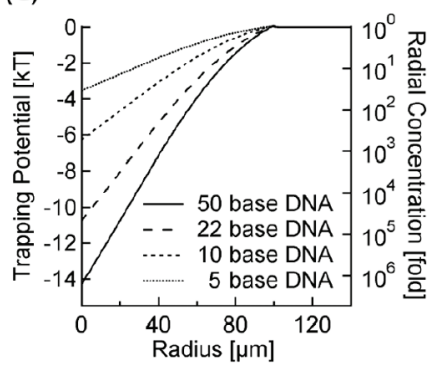

(d)

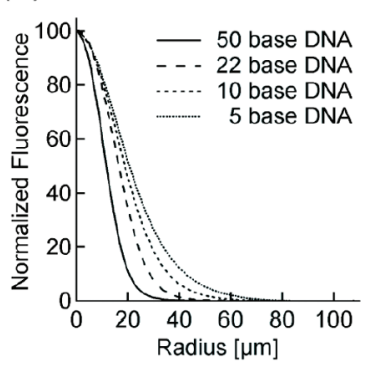

(e)

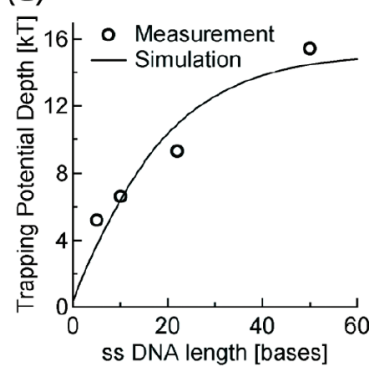

Figure 2. Theory. (a) Finite element simulation in an axial symmetry confirms the experimental observations and predicts a steady-state concentration of $\left(2 \times 10^{6}\right)$-fold for single-stranded 50 base DNA. Arrows represent the fluid flow. (b) Molecules in the trap are shuffled to the center within $3 \mathrm{~s}$ as shown by the time trace of the center concentration with $c \propto t^{1.2}$. The successively slower concentration increase $\left(c \propto t^{0.4}\right)$ refers to diffusion into the region of the trap. The simulation (solid line) confirms the experimental behavior (open circles) (c) Radial distribution of the simulated trapping potential for 5, 10, 22, and 50 base DNA. (d) Measured concentration distribution of the conveyor belt. (e) Measured trapping potential depths for the DNA oligomers (open circles) match finite element simulations (solid line).

of $8 k T$. The pressure difference from the fluid viscosity in a thinner chamber does not deform the chamber and is not limiting the flow speed. ${ }^{13}$ The thermal relaxation of such a chamber is on the order of $5 \mu \mathrm{s}$, still faster than the laser deflection. However faster laser deflections are hard to achieve, and the shown experiments are at the speed limit of acousto-optical deflectors. Therefore, although the optical driving is very attractive due to its flexibility, electrical surface heating might be useful to reach the physical limits of the molecular conveyor.

The presented approach complements and extends previous methods to manipulate and trap small objects. Cohen and Moerner showed a fluorescence feedback trap based on microstructured electrophoresis. ${ }^{3}$ This technique proved successful in trapping single molecules down to the size of a few nanometers. ${ }^{17}$ For this method, the position of a trapped object has to be obtained in real time. Optical tweezers ${ }^{1,18-20}$ have been employed to grab and move objects on the microscale under physiological conditions. However the trapping is restricted to the area of the optical focus at the 1 $\mu \mathrm{m}$ scale and limited to particle sizes not much smaller than $100 \mathrm{~nm}$ since the optical power for trapping scales cubically to the reciprocal diameter. ${ }^{18}$ In comparison, thermophoresis scales with the particle surface. ${ }^{8}$

To conclude, we show an optical conveyor belt for small molecules in bulk water. Unlabeled molecules with the size of $1.5 \mathrm{~nm}$ are transported directionally and accumulated against the Brownian motion to a single spot on the micrometer scale.

Materials and Methods. Chamber and Infrared Heating. A $2 \mu \mathrm{m}$ thin water layer is sandwiched between a bottom silicon wafer and a top sapphire window (thickness $170 \mu \mathrm{m}$, diameter $12 \mathrm{~mm}$ ). The silicon wafer (thickness $0.5 \mathrm{~mm}$, polished on both sides, $1 \mu \mathrm{m} \mathrm{SiO}_{2}$, MEMC Electronic
Materials Inc.) is coated on one side with a $60 \mathrm{~nm}$ chromium layer by vacuum evaporation followed by $60 \mathrm{~nm} \mathrm{SiO}_{2}$ from plasma-enhanced chemical vapor deposition. An infrared laser beam (RLD-5-1455, IPGLaser, $\lambda=1455 \mathrm{~nm}, 5 \mathrm{~W}$ ) is deflected by an acousto-optical deflector (AA.DTS.XY.100, Pegasus Optics) and focused from below into the water sheet with a long working distance lens $(f=40 \mathrm{~mm}$, LA1304-C, Thorlabs). The laser passes through the $\mathrm{Si}$ and $\mathrm{SiO}_{2}$ which are transparent to the used wavelength but heats the $\mathrm{Cr}$ layer by absorption. The color-coded area in Figure $1 \mathrm{~b}$ shows a finite element simulation of the water temperature in a cross section of the chip. The sapphire window at the top of the fluid sheet has a high thermal conductivity and cools the water to less than $10 \%$ of the temperature at the bottom, resulting in a steep temperature gradient in the $z$ direction.

The solution used in Figures 1, 2, and $3 b$ consists of 1 $\mu \mathrm{M}$ single-stranded DNA labeled with the fluorescent dye HEX at the 5' end (Biomers, Ulm, Germany), dissolved in $10 \mathrm{mM}$ TRIS buffer at $\mathrm{pH}$ 10.4. Surface interactions reduce the $\mathrm{pH}$ to about $7-8$. The diffusion coefficients are measured for $50,22,10$, and 5 base DNA by evaluating the back diffusion after thermophoretic depletion ${ }^{8}$ and confirm free diffusion of DNA. For extrapolation, the diffusion coefficients of DNA were fitted phenomenologically with the power function $D=a_{1}(L+2)^{-a_{2}}$, where $L$ is the number of bases, $a_{1}=1020 \mu \mathrm{m}^{2} / \mathrm{s}$, and $a_{2}=0.75$. We assume an offset of two bases which have a similar diffusion coefficient than the HEX label. $S_{\mathrm{T}}$ values are expected to scale proportional with $L^{1 / 2}+$ constant. ${ }^{8}$ The Soret coefficient of 50 base singlestranded DNA results in $S_{\mathrm{T}}=0.006 \mathrm{~K}^{-1}+0.009 \mathrm{~K}^{-1} L^{1 / 2}$, with an offset assigned to the HEX label, measured to 0.006 $\mathrm{K}^{-1}$.

Imaging and Scanning. Imaging is provided with an upright fluorescence microscope (Axiotech Vario, Zeiss) with 

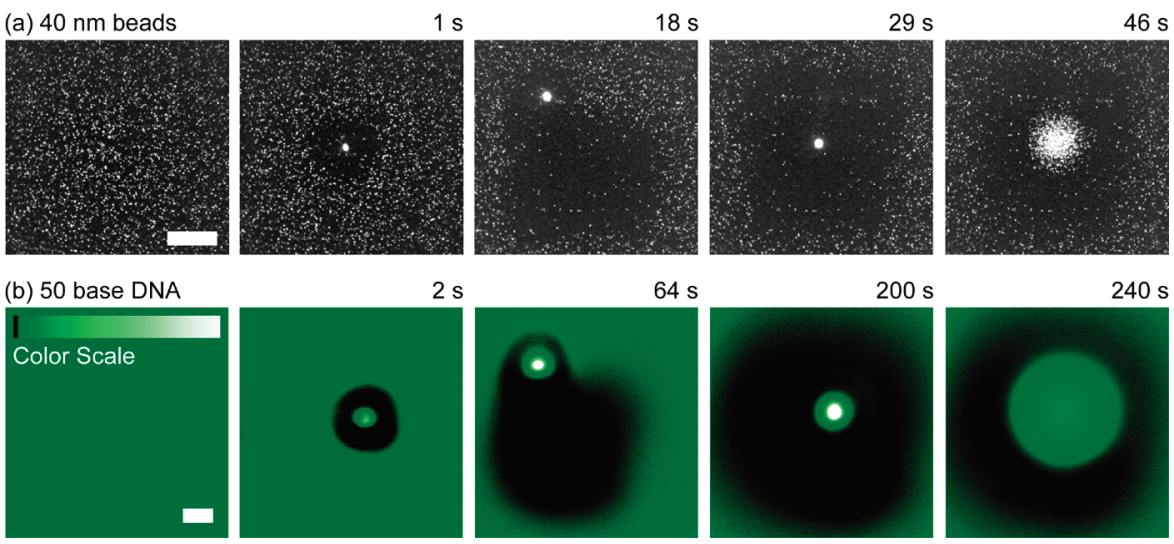

Figure 3. Moving trap. (a) Since the conveyor belt is driven entirely optically, it can be moved arbitrarily through the solution to collect polystyrene beads with a diameter of $40 \mathrm{~nm}$. After the trap has switched off, the particles diffuse freely. (b) The same processing works for single-stranded 50 base DNA. The color scale is highly nonlinear to visualize both the high concentration in the trapping center and the comparable low contrast between the outside concentration and the depleted area. The scale bars are $100 \mu \mathrm{m}$. (See both movies in Supporting Information.)
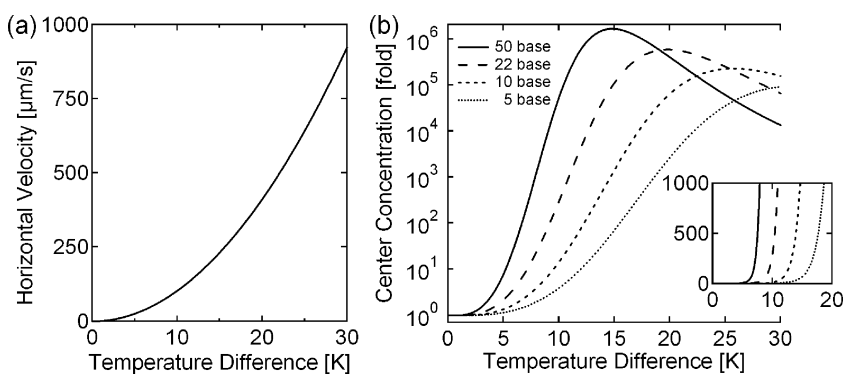

Figure 4. Simulated temperature dependence of the conveyer. (a) The optothermal driving increases the flow velocity of the conveyor flow quadratically with the applied temperature. (b) As a result, the accumulation efficiency is not an exponential function of temperature but can even drop for higher temperatures since the flow is too fast to allow diffusion to optimally equilibrate the thermophoretic concentration profile of the molecules across the chamber. Inset: for the same reason, accumulation raises hyperexponentially at small temperature differences as shown in the linear plot.

a CCD camera (Cascade II 512, Photometrics) and a $20 \times$ objective (UPlanSApo NA $=0.75$, Olympus), illuminated with an LED (LXHL-LD3C, Luxeon). Chamber-averaged temperature is imaged, using the temperature sensitive dye Cy5 $(170 \mu \mathrm{M}$ Cy5 in $150 \mathrm{mM} \mathrm{NaCl}$ and $15 \mathrm{mM}$ sodium citrate) with an exposure time of $50 \mathrm{~ms}$ straight after the onset of the scanning pattern before mass diffusion sets in, which traps the temperature dye. We find an average increase in temperature of $7.5 \mathrm{~K}$. With the simulated shape of the temperature profile, we can infer that the warm side is heated $15 \mathrm{~K}$ over room temperature. Both the size $(1 \mathrm{~cm}$ vs 200 $\mu \mathrm{m}$ of the trap) and high thermal conductivity (149 W/(m $\mathrm{K})$ vs $0.6 \mathrm{~W} /(\mathrm{m} \mathrm{K})$ of water) of the silicon substrate ensure thermal coupling to room temperature. Cooling of the setup would allow to decrease the maximal temperature in the trap. The laser spot is defocused to a diameter of $d_{1 / e}=70 \mu \mathrm{m}$ and scanned in radial direction from a radius of $100 \mu \mathrm{m}$ toward the center point along 64 different angles with a frequency of $8 \mathrm{kHz}$ per line.

Acknowledgment. The authors thank Ann Fornof for reading the manuscript and Ingmar Schön for chip preparations. The work was supported by the Emmy-Noether Program of the Deutsche Forschungsgemeinschaft (DFG), the LMU Innovative Initiative Functional NanoSystems (FUNS), and the Excellence Cluster NanoSystems Initiative Munich (NIM).

Supporting Information Available: Two videos of moving traps. This material is available free of charge via the Internet at http://pubs.acs.org.

\section{References}

(1) Ashkin, A. Phys. Rev. Lett. 1970, 24, 156.

(2) Rudhardt, D.; Bechinger, C.; Leiderer, P. Phys. Rev. Lett. 1998, 81, 1330 .

(3) Cohen, A. E.; Moerner, W. E. Proc. Natl. Acad. Sci. U.S.A. 2006, 103,4362 .

(4) Hertlein, C.; Helden, L.; Gambassi, A.; Dietrich, S.; Bechinger, C. Nature 2008, 451, 172.

(5) Soret, C. Arch. Sci. 1879, 3, 48.

(6) Rauch, J.; Köhler, W. Phys. Rev. Lett. 2002, 88, 185901.

(7) Brenner, H. Phys. Rev. E 2006, 74, 036306.

(8) Duhr, S.; Braun, D. Proc. Natl. Acad. Sci. U.S.A. 2006, 103, 19678.

(9) Braun, D.; Libchaber, A. Phys. Rev. Lett. 2002, 89, 188103.

(10) Duhr, S.; Braun, D. Phys. Rev. Lett. 2006, 97, 038103.

(11) Yariv, E.; Brenner, H. Phys. Fluids 2004, 16, L95.

(12) Weinert, F. M.; Kraus, J. A.; Franosch, T.; Braun, D. Phys. Rev. Lett. 2008, 100, 164501.

(13) Weinert, F. M.; Braun, D. J. Appl. Phys. 2008, 104, 104701.

(14) Bou-Ali, M. M.; Ecenarro, O.; Madariaga, J. A.; Santamaria, C. M.; Valencia, J. J. J. Phys.: Condens. Matter 1998, 10, 3321.

(15) Baaske, P.; Weinert, F. M.; Duhr, S.; Lemke, K. H.; Russell, M. J.; Braun, D. Proc. Natl. Acad. Sci. U.S.A. 2007, 104, 9346.

(16) Clusius, K.; Dickel, G. Naturwissenschaften 1938, 26, 546.

(17) Cohen, A. E.; Moerner, W. E. Phys. Rev. Lett. 2007, 98, 116001.

(18) Ashkin, A.; Dziedzic, J. M.; Bjorkholm, J. E.; Chu, S. Opt. Lett. 1986, $11,288$.

(19) Ashkin, A. Proc. Natl. Acad. Sci. U.S.A. 1997, 94, 4853.

(20) Block, S. M.; Goldstein, L. S. B.; Schnapp, B. J. Nature (London) 1990, 348, 348.

NL902503C 